\title{
Opioid Taper is Associated with Improved Experimental Pain Tolerance in Patients with Chronic Pain: An Observational Study
}

\author{
Peggy Compton (D) - Olivia M. Halabicky · Subhash Aryal • \\ Ignacio Badiola
}

Received: November 5, 2021 / Accepted: December 10, 2021 / Published online: January 12, 2022

(c) The Author(s) 2022

\begin{abstract}
Introduction: The degree to which opioid-induced hyperalgesia contributes to the pain experience of patients with chronic pain remains relatively undescribed. The objective of this pilot study was to determine if experimental pain responses improve in patients with chronic pain as they undergo a planned opioid taper.

Methods: This was a prospective observational study. Seven patients with chronic neuropathic pain on at least $120 \mathrm{mg}$ morphine equivalents/day were enrolled. The participants were followed over the course of an individualized opioid taper to a lower dose. Measures of experimental pain sensitivity, including indicators of central pain modulation, were collected on a biweekly basis; in addition, measures of function and quality of life were collected
\end{abstract}

Supplementary Information The online version contains supplementary material available at https:// doi.org/10.1007/s40122-021-00348-8.

P. Compton $(\varangle) \cdot$ O. M. Halabicky · S. Aryal Family and Community Health, School of Nursing, University of Pennsylvania, Claire M. Fagin Hall, Room 402, 418 Curie Blvd, Philadelphia, PA 191044217, USA

e-mail: pcompton@nursing.upenn.edu

I. Badiola

Perelman School of Medicine, University of

Pennsylvania, Philadelphia, PA, USA monthly. The effect of opioid taper on pain responses and functional outcomes over time were examined using longitudinal mixed-effects regression modeling and general linear regression modeling with regularization as a function of baseline dose, end dose, and taper rate.

Results: In this small sample of patients undergoing highly individualized and variable opioid taper, the opioid taper was significantly associated with improved pain responses to the cold-pressor test, with the pain threshold on average increasing by $1.14 \mathrm{~s}$ every 6 weeks $(p=0.0084,95 \%$ confidence interval $[\mathrm{CI}]$ for 6-week change 0.3039-2.0178) and pain tolerance on average increasing by $2.87 \mathrm{~s}$ every 6 weeks $(p=0.0026,95 \%$ CI for 6-week change 1.02-4.7277). Taper-related changes in central pain modulation were not observed, although conditioned modulation trended toward improvement by the completion of opioid taper. Similarly, no declines in function and quality of life were observed with the opioid taper, suggesting stability despite decreased opioid dose.

Conclusions: Opioid taper was associated with improvements in experimental pain responses without a decline in function and quality of life, suggestive of diminished opioid-induced hyperalgesia in this clinical sample.

Trial Registration: ClinicalTrials.gov identifier, NCT03912298. 
Keywords: Chronic pain; Cold-pressor test; Opioid; Quantitative sensory testing; Taper

\section{Key Summary Points}

Why carry out this study?

It is estimated that between 5 and 8 million Americans use opioids daily for chronic pain management however, it is increasingly clear that outcomes are often poorer for patients on opioid therapy.

A theorized explanation for poorer outcomes for patients on opioid therapy is the phenomenon of opioid-induced hyperalgesia.

We hypothesized that patients undergoing opioid taper would have improved experimental pain responses over time.

What was learned from the study?

Opioid taper is associated with improved pain responses to the cold-pressor test.

Opioid taper is associated with improvements in experimental pain responses without a decline in function and quality of life, suggesting diminished opioid-induced hyperalgesia in this clinical sample

\section{INTRODUCTION}

Chronic pain impacts approximately $20 \%$ of U.S. adults, and $8 \%$ of U.S. adults have highimpact chronic pain [1], with prevalence expected to increase as the population ages. In well-intended and industry-driven efforts to provide relief to chronic pain sufferers, the prescription of opioids has increased dramatically since the turn of the century, such that it has been estimated that between 5 and 8 million Americans use opioids daily for chronic pain management [2]. However, prescription opioid therapy for chronic pain is not an evidence-based intervention. In fact, as evaluation data accumulate, it is increasingly clear that outcomes are often poorer for patients on opioid therapy and that improvements can be appreciated when patients are tapered off the analesics $[3,4]$. In the midst of an "epidemic of prescription drug abuse" [5], it is critical that opioid prescription practices be evidence-based and delivered "in the best possible manner that maximizes effectiveness and minimizes harm" [6].

A theorized explanation for poorer outcomes for patients on opioid therapy is the phenomenon of opioid-induced hyperalgesia $(\mathrm{OIH})$ [7]. Well-demonstrated in animals [8] and inferred in opioid-maintained patients with opioid use disorder [9], ongoing opioid use results in increased sensitivity to pain, which, in the case of the patient with chronic pain, may interfere with desired pain relief outcomes. However, the relationship between opioid taper and $\mathrm{OIH}$ has received little empirical attention, such that the degree to which decreasing opioid provision improves pain sensitivity is not clear. Evidence that prescription opioids make the pain experience worse for patients with chronic pain would provide strong support for the opioid-sparing approaches promulgated by the U.S. Centers for Disease Control and Prevention (CDC) [10] and provide novel data on which to develop evidence-based guidelines.

Studying the direct effects of an opioid taper on pain responses in chronic pain patients is challenging; to be successful, a taper must be gradual, and individualized to patient response and tolerability $[11,12]$. We designed an observational study to identify and characterize the direct effects of opioid taper on experimental pain responses in opioid-dependent patients with chronic pain to establish if, in fact, a notable effect size can be discerned. We hypothesized that subjects undergoing opioid taper will have improved experimental pain responses over time. In addition, we anticipated that improvements in pain sensitivity would result in concurrent improvements in functional outcomes. If supported, these findings would provide a foundation upon which to predict the efficacy of opioid taper in larger clinical samples of patients with chronic pain. 


\section{METHODS}

\section{Design}

An observational prospective design was utilized to measure the experimental pain responses and function of chronic pain patients on high-dose opioid therapy prior to and then regularly during a voluntary individualized opioid taper. Pain data were collected on a biweekly basis and functionality data were collected monthly, for up to 12 months. Due to the pilot nature of the study, our previous work demonstrating stability in pain responses in patients on high-dose opioid therapy over time (6 months) [9], and the clinical imperative of tapering patients to safer doses, we did not include a control group of patients maintained on high-dose therapy. Consistent with expert opinion in the field [13], a longitudinal withinsubject design was planned.

\section{Sample and Setting}

The convenience sample consisted of male and female adults with chronic pain undergoing opioid taper. Study inclusion criteria included: (1) being between the ages of 21-70 years; (2) having a documented chronic neuropathic non-malignant pain condition of at least 1 year duration; (3) on $\geq 120 \mathrm{mg}$ opioid morphine equivalents daily (MED) for at least 6 months; (4) having fully engaged in all prescribed nonopioid pain management treatments; and (5) otherwise in good physical and mental health. To control for variability related to the type of chronic pain, only patients determined to have neuropathic pain (as assessed by the validated Leeds Assessment of Neuropathic Symptoms and Signs pain scale) were included [14]. Having fully utilized all non-opioid medication and multimodal pain management approaches assured that no new pain management interventions would be introduced over the course of the taper.

Potential participants were excluded from participation if: (1) they met diagnostic criteria for an active substance use disorder other than nicotine; (2) were acutely psychotic, severely depressed, and/or in need of inpatient psychiatric treatment; (3) had a neurological or psychiatric illness that would affect pain responses, including anxiety disorders; or (4) had a history of heart disease, stroke, or a pacemaker or uncontrolled high blood pressure. Good cardiovascular health was stipulated to ensure subjects could tolerate the sympathetic responses associated with the cold-pressor pain induction procedures.

Participants were recruited from the large University of Pennsylvania Penn Pain Medicine Center. Recruitment occurred over a 19-month period, being slowed by the paucity of patients on high-dose opioid therapy willing to voluntarily undergo taper and the coronavirus epidemic. The study protocol was approved by the Institutional Review Board (IRB) of the University of Pennsylvania (Protocol \# 831447), and all subjects signed and demonstrated their comprehension of the IRB-approved consent form. Subjects were compensated for participation.

\section{Measures}

\section{Pain Responses}

The primary dependent variable, pain sensitivity, was measured using two highly reliable and valid pain induction techniques, the cold-pressor test (CPT) and quantitative sensory testing (QST), employing procedures consistent with those described in the literature [15-17]. The order of pain testing varied, and three aspects of the pain response associated with $\mathrm{OIH}$ were captured at each study session: evoked pain, temporal summation, and conditioned pain modulation, which map on the hypothesized peripheral, spinal and supra-spinal mechanisms of pain processing, respectively (see Electronic Supplementary Material for pain induction technique methods). Concurrent with pain testing, the presence of opioid withdrawal symptoms using the well-validated clinical opiate withdrawal scale (COWS) [18] was assessed. In addition, urine toxicology was collected to ensure that subjects are taking only their prescribed opioid at each pain data collection session. To account for the effects of disease progression on pain responses, the study 
physician evaluated subjects monthly for any evidence of disease worsening.

\section{Functional Outcomes}

To evaluate if opioid taper improves functional outcomes vis-à-vis improved pain responses, we used the PROMIS ${ }^{\circledR}$ (Patient-Reported Outcomes Measurement Information System) person-centered measures [19]. Specifically measured were functional and quality of life indicators from the physical health (pain intensity, pain interference, physical function), mental health (life satisfaction), and social health (ability to participate in social roles and activities) domains. PROMIS scores were assessed for change from baseline and in relationship to changes in experimental pain assay performance.

\section{Procedures}

In light of the demonstrated health risks associated with daily opioid doses $>90 \mathrm{mg}$ MED [20-23], current practice at the Penn Pain Medicine Center is to taper all chronic pain patients down to or below this MED and to stabilize them on the lowest effective dose as agreed upon by the patient and prescriber. The actual rate of taper varies with tolerability and availability of dosages of opioid, but averages $10-15 \%$ decrease/month.

Subject recruitment was accomplished via IRB-approved flyers made available and posted at the Pain Medicine Center, and treatment clinicians were oriented to identify potential subjects. Patients expressing interest in study participation met with a trained project coordinator for screening; if the patient met all inclusion and no exclusion criteria, informed consent was obtained in a private research area at the Center. Study data collection began immediately following consent, with the collection of urine toxicology, chronic pain and opioid use history, and baseline pain testing. Study sessions were scheduled immediately prior to or following regular clinic visits as possible to minimize subject burden. Subjects were instructed to ingest no caffeine or nicotine for $1 \mathrm{~h}$ prior to each session. Opioid withdrawal score and urine sample for toxicology were collected immediately prior to pain testing to verify absence of opioid withdrawal and compliance with opioid treatment. All study data were collected and managed using REDCap (Research Electronic Data Capture) data capture tools hosted at the School of Nursing at the University of Pennsylvania [24].

\section{Data Analysis}

Preliminary analyses included generating descriptive statistics for all baseline measures to characterize the sample, including measures of central tendency and variation for continuous variables; for dichotomous/categorical variables, measures included frequencies and percentages. Distributional properties were examined to determine if variance stabilizing or normalizing transformations were required. Non-inferential interim analyses were performed to ensure data collection and archiving procedures were operating correctly. Outliers were assessed by visual inspection and checked for accuracy. Normality was assessed using Shapiro-Wilk tests.

The effect of opioid taper on changes in pain responses (CPT, QST) and functional outcomes (PROMIS domains) over time were examined using longitudinal mixed-effects regression modeling and general linear regression modeling with regularization as a function of baseline dose, end dose, and taper rate [25]. These longitudinal profiles were examined using a linear mixed-effects framework with SAS Proc Mixed [26] in which all available data collected during follow-up were used. Mixed models can account for the correlation between repeated measures and also allow for heterogeneity between subjects at baseline.

Separate mixed-effects regression models were generated for each of the continuous outcome measures of interest (pain responses, functional outcomes). Random intercepts were modeled to represent the participant-level deviation from the fixed-effect slope over time and intercept, respectively. Maximum likelihood estimation was used and independence covariance structure was assumed. The Akaike information criterion was used to evaluate 
overall model fit and to select the best-fitting longitudinal change pattern. [27].

\section{RESULTS}

A total of seven patients with neuropathic pain and on opioid therapy were enrolled in the study, the majority of whom were middle-aged, female and White (see Table 1). The median (interquartile range [IQR]) opioid MED dose at baseline was 270 (267) $\mathrm{mg}$ and decreased to 127.5 (73) $\mathrm{mg}$ at completion of the taper (see Table 2). The mean (standard deviation [SD]) opioid MED dose at baseline was 291.9 (182.6) $\mathrm{mg}$ with a minimum of $127 \mathrm{mg}$ and maximum of $645 \mathrm{mg}$. The average MED dose following the taper period was 258.0 (164.2) $\mathrm{mg}$ with a minimum of $90 \mathrm{mg}$ and a maximum of $615 \mathrm{mg}$, representing an approximately 12\% reduction over time. Median (IQR) chronic pain score on the visual analogue scale (VAS) was 98 (10) at baseline and 90 (0) at the taper completion.

Average (SD) chronic pain score on the VAS at baseline was 85.4 (29.2) on a scale of 0-100, which decreased to 74.1(37.3) by the end of the taper, representing a $16 \%$ improvement following the taper. Improvements in median cold pressor pain threshold, pain tolerance, and PROMIS scores were evident following the taper (Table 2). The taper period ranged from 6 to 102 weeks; although highly individualized, the

Table 1 Sample characteristics ( $n=7$ patients)

\begin{tabular}{ll}
\hline Characteristics of patients & Values \\
\hline Race, $n(\%)$ & \\
White & $6(85.71 \%)$ \\
Other & $1(14.29 \%)$ \\
Sex, $n(\%)$ & \\
Female & $5(71.3 \%)$ \\
Age (years) & $61(27)$ \\
Duration of taper (weeks) & $17.5(21.5)$ \\
\hline
\end{tabular}

Data are presented as the median with the interquartile range (IQR) in parentheses unless indicated otherwise rate of taper across participants averaged $4.3 \mathrm{mg}$ MED every 2 weeks. Urine toxicology results across the tapers showed the presence of prescribed opioids and, in one patient, prescribed benzodiazepine, but were negative for all other substances of abuse, including cannabis. Figure 1 shows the individual responses of each subject.

Prior to the pain testing sessions, participants presented with either no or mild opioid withdrawal symptoms; there was no significant change in average withdrawal symptoms during the taper period (Estimate $=-0.0253,0.0170$, $p=0.1392$ ). Using a linear mixed-effects model with a random intercept we determined that the opioid taper significantly improved pain responses to the cold-pressor test, with the pain threshold on average increasing by $1.14 \mathrm{~s}$ every 6 weeks $(p=0.0084 ; 95 \%$ confidence interval [CI] for 6-week change 0.3039-2.0178) and tolerance increasing by $2.87 \mathrm{~s}$ every 6 weeks $(p=0.0026 ; \quad 95 \% \quad$ CI for 6-week change 1.02-4.7277). Significant differences over the course of the opioid taper on temporal summation and conditioned pain modulation were not observed; temporal summation responses tended to remain unchanged over time, and conditioned pain modulation tended to decrease over time from baseline $(-0.1428)$ to taper completion $(-0.40)$ (difference 35\%). Concurrent with the opioid taper, indicators of functionality, perceived life satisfaction, and the ability to participate in social roles and activities did not show any statistically significant changes with opioid taper.

\section{DISCUSSION}

In this small observational study of a wellcharacterized sample of men and women with chronic neuropathic pain and receiving highdose opioid therapy ( $\geq 120 \mathrm{mg}$ MED), significant improvements in experimental cold-pressor pain threshold and tolerance were associated with an individualized taper such that, on average, pain threshold increased by $50.5 \%$ and tolerance increased by $75 \%$ upon taper completion. Noted improvements in coldpressor pain responses specifically support a 
Table 2 Pain and function prior to and following taper

\begin{tabular}{lcc}
\hline & Baseline & Final \\
\hline MED (mg) & $270(267)$ & $127.5(73)$ \\
Cold-pressor pain threshold (s) & $12.75(10.35)$ & $7.78(5.59)$ \\
Cold-pressor pain tolerance (s) & $17.23(19.03)$ & $16.64(74.48)$ \\
Chronic Pain Severity (average; 0-100) & $98(10)$ & $90(0)$ \\
PROMIS score & $110(42)$ & $110.5(54)$ \\
\hline
\end{tabular}

Data in table are presented as the median with the IQR in parentheses

MED Morphine equivalents per day, PROMIS Patient-Reported Outcomes Measurement Information System

reversal of $\mathrm{OIH}$, in that thermal experimental pain responses (as opposed to electrical or pressure pain) have been demonstrated to be the most reliable and robust indicator of OIH in clinical populations [28, 29]. Further, the approximately $12 \%$ decrease in daily opioid dose achieved during the taper resulted in a $16 \%$ improvement in daily average severity of chronic pain, suggesting that the taper was associated with a reduction in the effects of $\mathrm{OIH}$ on the chronic pain experience for these patients.

Changes in central pain modulation (i.e., temporal summation, conditioned pain modulation) related to opioid taper were less evident in this sample. This is somewhat inconsistent with the extant literature, although none of the existing studies evaluated within-individual changes, only differences between persons on versus not on opioid therapy. For example, Chen and colleagues [17] found evidence of exacerbated temporal summation to thermal stimulation in patients with chronic pain and on opioid therapy in comparison to those not taking opioids, whereas no patterned changes in temporal summation were noted in the current sample. However, decreased conditioned pain modulation to thermal pain noted by Ram and colleagues [30] in patients with chronic pain and on opioid therapy versus those on non-opioid therapy mirror the trend noted in the current study, supporting improved conditioned pain modulation following taper (albeit not significantly so). The work of Edwards and colleagues [31] suggest that negative affect may confound the noted relationship between opioid use and pain modulation, such that patients with chronic pain and on opioid therapy with high levels of negative affect experienced less conditioned pain modulation to cold pain and appeared more hyperalgesic than those with low levels of negative affect. The small sample size in the current study precluded examination of the role of affect in taper outcomes; however, improved modulation noted in the patients following taper map on the conclusions of Edwards who suggest a negative correlation between $\mathrm{OIH}$ and conditioned pain modulation in patients with chronic pain and on opioid therapy.

Not noted in the current investigation were improvements nor worsening in function or quality of life with opioid taper. Despite being in contrast with findings from several systematic reviews which suggested improvements in these domains for patients with chronic pain when opioids are tapered or discontinued [2, 4], these findings support more recent work demonstrating that with opioid taper, function and quality of life remain unchanged and do not worsen. For example, Laigaard and colleagues [32] show that an opioid taper from an average of $80 \mathrm{mg}$ MED to $19 \mathrm{mg}$ MED brought no improvement in health-related quality of life or worsened symptoms of depression or anxiety, despite minor improvements in cognitive function. Similarly, DiBenedetto and colleagues reported no increase in disability with significant opioid dose reductions [33], and in a sample of veterans with chronic pain, the effect 
$\mathbf{a}$

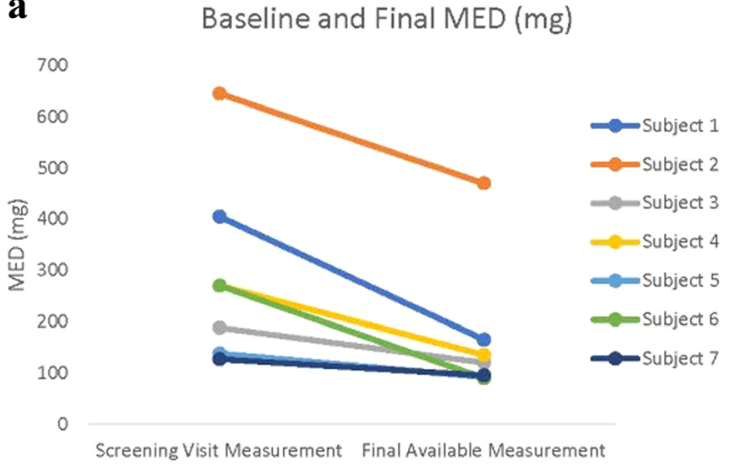

c Cold-pressor pain tolerance(sec)
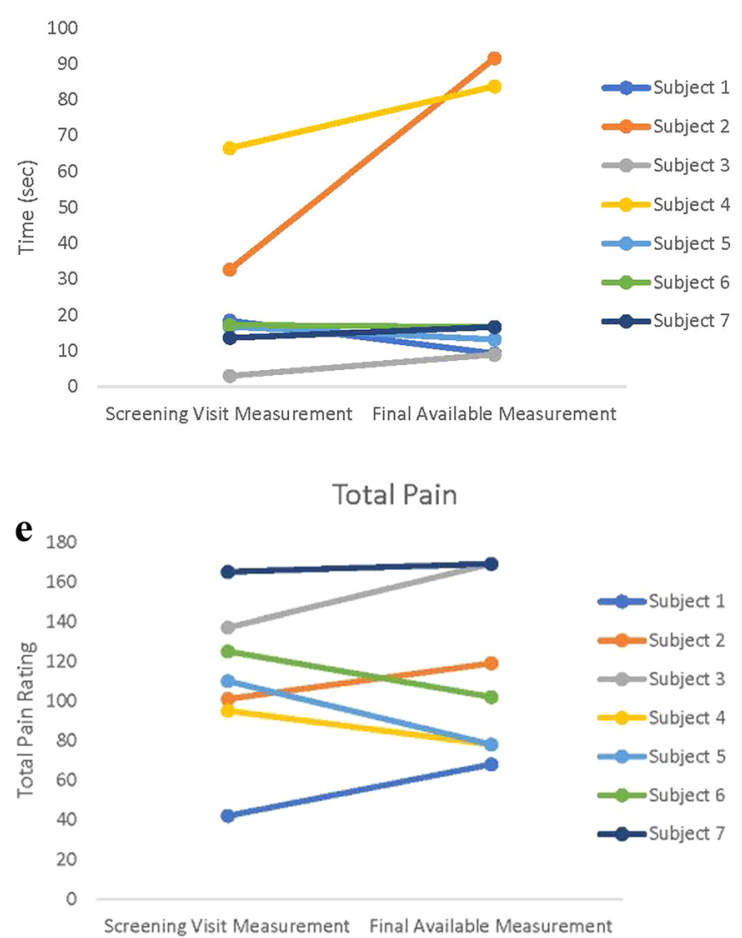

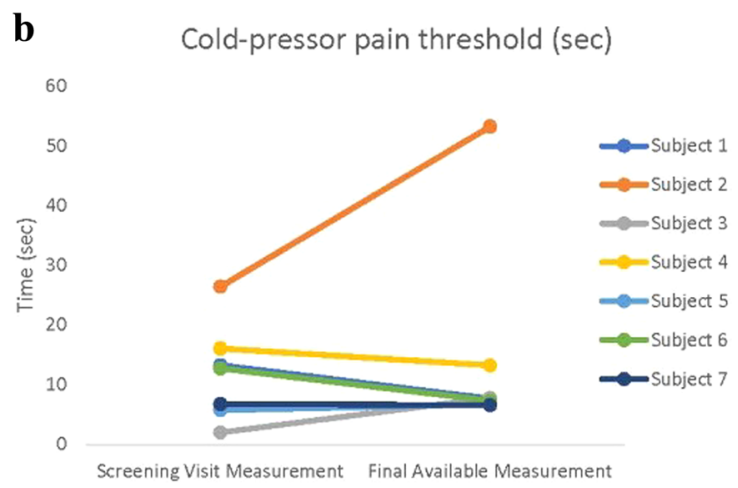

d Chronic Pain Severity

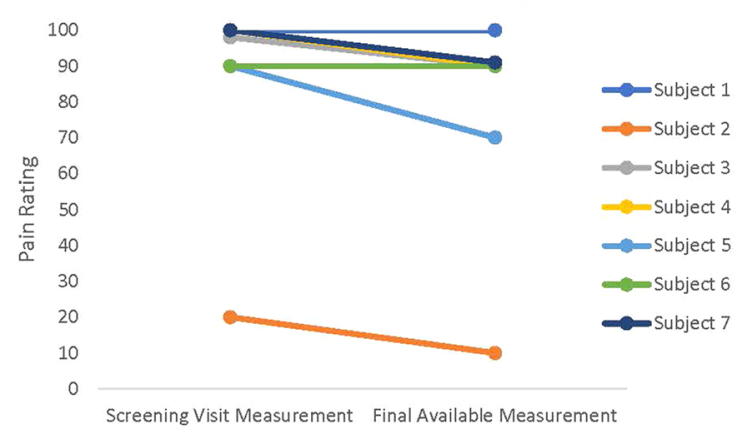

Taper Period in Weeks

\section{f}

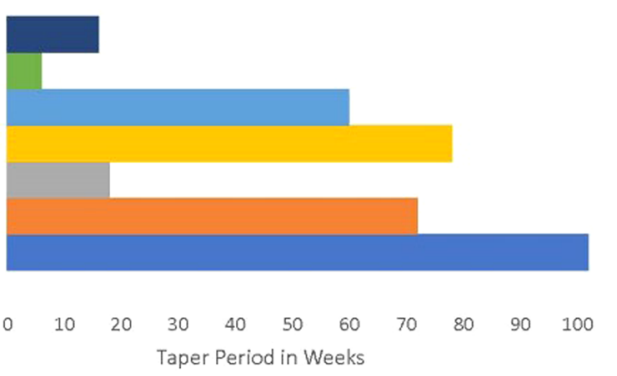

- Subject 7
- Subject 6
- Subject 5
- Subject 4
- Subject 3
- Subject 2
- Subject 1

110

Fig. 1 Change in individual patient responses over the opioid taper. a MED, $\mathbf{b}$ cold-pressor pain threshold, $\mathbf{c}$ cold-pressor pain tolerance, $\mathbf{d}$ chronic pain severity, e PROMIS score, $\mathbf{f}$ length of taper. $M E D$ Morphine equivalents per day

of interdisciplinary pain treatment achieved comparable efficacy for both patients not taking opioids and those tapered off their opioids [34]. Although a 2017 systematic review of patient outcomes following a dose reduction or discontinuation of long-term opioid therapy reported improvements in pain severity, function, and quality of life, the quality of this evidence was deemed to be of very low quality [4]. In fact, a more recent rapid review of the literature found that pain severity either remain unchanged or improved with opioid taper; however, improvements in quality of life and functionality were not consistently observed, and much of the evidence remains at the level of low quality. In addition, more recent data suggest harms associated with opioid taper, including increased risk of overdose and suicidal ideation [35]. Despite the opioid taper in the current sample being modest, the overall 
clinical implications of these findings are consistent with the literature finding that function and quality of life remain stable and do not worsen with opioid dose reduction.

It should be noted that the increases in experimental cold-pressor pain threshold and tolerance, albeit statistically significant, are relatively small. Concurrent improvements in pain severity ratings noted in the current study $(16 \%)$ approach the percentage change of patient-defined minimal clinically important improvement in chronic musculoskeletal pain or low back pain ( $\geq 15 \%$ ) [36], providing evidence that these changes in experimental pain sensitivity may translate to improvements in chronic pain severity. However, a lack of improvement in pain-related function and quality of life, as well as mixed and nonsignificant effects on opioid-related pain modulation activities, should temper clinician motivation to taper a given patient's opioid therapy to result in improved pain outcomes. As cautioned by Mackey and colleagues [35] and others $[37,38]$, evidence for the benefits versus harms associated with opioid taper remains mixed, and, as promulgated by a recent American Academy of Pain Medicine Foundation consensus panel, these should not be attempted in patients with chronic pain without adopting "a patient-centered, integrated, comprehensive treatment models employing a biopsychosocial perspective" (p. 2155 [39]).

\section{Limitations}

There are multiple weaknesses associated with this pilot study that limit interpretation and application to the clinical management of patients with chronic pain. Firstly, the study was observational, and patients were recruited based on convenience and relied on self-selection; causality cannot be assured with this nonexperimental design and the findings may not be generalizable to the larger population of patients with chronic pain attempting opioid taper. The sample was quite small, precluding evaluation of the effects of patient characteristics, pain characteristics, and taper characteristics on outcomes assessed; further utilization of mean values in the analysis with so few subjects limits interpretation. The absolute amount of opioid taper was relatively small, and greater gains in pain response, function, and quality of life may have been appreciated with a greater decrease in opioid dosage. Finally, the pain testing was repeated multiple times over the course of the taper, so participants may have developed some habituation to the noxious stimulus, resulting in improved pain responses over time. However, pain testing was separated by 2-week intervals to minimize this learning effect and improvements were not noted in all pain parameters, arguing against this.

\section{CONCLUSION}

In this pilot study of a small, well-characterized sample of patients with chronic pain, an individualized opioid taper was associated with significant improvements in threshold and tolerance to experimental cold-pressor pain. These findings, in combination with a trend toward perceived decrease in average severity of chronic pain following opioid taper, argue that OIH may contribute to the pain experience of patients with chronic pain. Notable was that decreases in opioid prescription did not worsen function or quality of life in the sample. However, the benefits associated with these improvements in experimental pain sensitivity are preliminary and cannot alone support opioid taper in all patients with chronic pain. The benefits of opioid therapy for an individual patient must be considered relative to risks, and the apparent minimal contribution of OIH to the chronic pain experience may not warrant taper to meaningfully improve pain outcomes.

\section{ACKNOWLEDGEMENTS}

The authors thank all study participants for their involvement in the study.

Funding. This work was supported by the National Institutes on Drug Abuse (R21 DA046364 [P.C.]), the National Institute of 
Nursing Research (R21 NR019047 [P.C.] F31 NR019527 [OMH]), and the National Institute of Environmental Health Sciences (T32 ES007062 [OMH]). The Journal's Rapid Service Fee was provided by the Van Ameringen Endowment (P.C.).

Medical Writing, Editorial, and Other Assistance. The authors have no medical writing, editorial, and other assistance to acknowledge.

Authorship. All named authors meet the International Committee of Medical Journal Editors (ICMJE) criteria for authorship for this article, take responsibility for the integrity of the work as a whole, and have given their approval for this version to be published.

Author Contributions. Peggy Compton and Ignacio Badiola contributed to the study conception and design. Material preparation, data collection, and data analysis were performed by Olivia Halabicky and Subhash Aryal. The first draft of the manuscript was written by Peggy Compton, and all authors commented on previous versions of the manuscript. All authors read and approved the final manuscript.

Disclosures. Peggy Compton, Olivia Halabicky, Subhash Aryal, and Ignacio Badiola declare that they have nothing to disclose. Olivia Halabicky is now affiliated with the School of Public Health at the University of Michigan, Ann Arbor MI, USA.

Compliance with Ethics Guidelines. The study protocol was approved by the Institutional Review Board (IRB) of the University of Pennsylvania (Protocol \# 831447), and all subjects signed and demonstrated comprehension of the IRB-approved informed consent form. The study was performed in accordance with the Helsinki Declaration of 1964, and its later amendments. No identifying information is contained in the manuscript.

Data Availability. The datasets used and/or analyzed during the current study available from the corresponding author on reasonable request.

Open Access. This article is licensed under a Creative Commons Attribution-NonCommercial 4.0 International License, which permits any non-commercial use, sharing, adaptation, distribution and reproduction in any medium or format, as long as you give appropriate credit to the original author(s) and the source, provide a link to the Creative Commons licence, and indicate if changes were made. The images or other third party material in this article are included in the article's Creative Commons licence, unless indicated otherwise in a credit line to the material. If material is not included in the article's Creative Commons licence and your intended use is not permitted by statutory regulation or exceeds the permitted use, you will need to obtain permission directly from the copyright holder. To view a copy of this licence, visit http://creativecommons.org/licenses/by$\mathrm{nc} / 4.0 /$.

\section{REFERENCES}

1. Dahlhamer J, Lucas J, Zelaya C, et al. Prevalence of chronic pain and high-impact chronic pain among adults-United States, 2016. MMWR Morb Mortal Wkly Rep. 2018;67:1001-6. https://doi.org/10. 15585/mmwr.mm6736a2.

2. Chou R, Turner JA, Devine EB, et al. The effectiveness and risks of long-term opioid therapy for chronic pain: a systematic review for a National Institutes of Health Pathways to Prevention Workshop. Ann Intern Med. 2015;162:276-86.

3. Dowell D, Haegerich TM. Changing the conversation about opioid tapering. Ann Intern Med. 2017;167(3):208-9. https://doi.org/10.7326/M171402 .

4. Frank JW, Lovejoy TI, Becker WC, et al. Patient outcomes in dose reduction or discontinuation of long-term opioid therapy: a systematic review. Ann Intern Med. 2017;167(3):181-91. https://doi.org/ 10.7326/M17-0598.

5. Executive Office of the President of the United States. Epidemic: Responding to America's prescription drug abuse crisis. 2011. https://www. whitehouse.gov/sites/default/files/ondcp/policy- 
and-research/rx_abuse_plan.pdf. Accessed 17 Aug 2021.

6. Reuben DB, Alvanzo AAH, Ashikaga T, et al. National Institutes of Health Pathways to Prevention Workshop: the role of opioids in the treatment of chronic pain. Ann Intern Med. 2015;162(4): 295-300.

7. Brush DE. Complications of long-term opioid therapy for management of chronic pain: the paradox of opioid-induced hyperalgesia. J Med Toxicol. 2012;8(4):387-92.

8. Angst MS, Clark JD. Opioid-induced hyperalgesia: a qualitative systematic review. Anesthesiology. 2006;104(3):570-87.

9. Compton P, Canamar C, Hillhouse $\mathrm{M}$, et al. Hyperalgesia in heroin dependent patients and the effects of opioid substitution therapy. J Pain. 2012;13(4):401-9.

10. Powell D, Haegerich TM, Chou R. CDC guideline for prescribing opioids for chronic pain-United States, 2016. MMWR Recomm Rep. 2016;65(1): $1-49$.

11. Frank JW, Levy C, Matlock DD, et al. Patients' perspectives on tapering of chronic opioid therapy: a qualitative study. Pain Med. 2016;17(10):1838-47.

12. Berna C, Kulich RJ, Rathmell JP. Tapering long-term opioid therapy in chronic noncancer pain: evidence and recommendations for everyday practice. Mayo Clin Proc. 2015;90(6):828-42.

13. Mao J. Clinical diagnosis of opioid-induced hyperalgesia. Reg Anesth Pain Med. 2015;40(6):663-4. https://doi.org/10.1097/AAP.0000000000000317.

14. Morgan KJ, Anghelescu DL. A review of adult and pediatric neuropathic pain assessment tools. Clin J Pain. 2017;33:844e52.

15. Compton $\mathrm{P}$, Wasser TE, Cheatle M. Increased experimental pain sensitivity in chronic pain patients who developed opioid use disorder. Clin J Pain. 2020;36:667-74. https://doi.org/10.1097/AJP. 0000000000000855.

16. Gehling J, Mainka T, Vollert J, et al. Short-term testretest-reliability of conditioned pain modulation using the cold-heat-pain method in healthy subjects and its correlation to parameters of standardized quantitative sensory testing. BMC Neurol. 2016;16:125. https://doi.org/10.1186/s12883-0160650-z.

17. Chen L, Malarick C, Seefeld L, et al. Altered quantitative sensory testing outcome in subjects with opioid therapy. Pain. 2009;143:65-70.
18. Tompkins DA, Bigelow GE, Harrison JA, et al. Concurrent validation of the Clinical Opiate Withdrawal Scale (COWS) and single-item indices against the Clinical Institute Narcotic Assessment (CINA) opioid withdrawal instrument. Drug Alcohol Depend. 2009;105(1-2):154-9.

19. Cella D, Hahn EA, Jensen SE, et al. Patient-reported outcomes in performance measurement. Research Triangle Park: RTI Press; 2015.

20. Bohnert AS, Valenstein M, Bair MJ, et al. Association between opioid prescribing patterns and opioid overdose-related deaths. JAMA. 2011;305(13): 1315-21. https://doi.org/10.1001/jama.2011.370.

21. Gomes T, Mamdani MM, Dhalla IA, et al. Opioid dose and drug-related mortality in patients with nonmalignant pain. Arch Intern Med. 2011;171(7): 686-91. https://doi.org/10.1001/archinternmed. 2011.117.

22. Gomes T, Redelmeier DA, Juurlink DN, et al. Opioid dose and risk of road trauma in Canada: a population-based study. JAMA Intern Med. 2013;173(3): 196-201. https://doi.org/10.1001/2013. jamainternmed.733.

23. Zedler B, Xie L, Wang L, et al. Risk factors for serious prescription opioid-related toxicity or overdose among Veterans Health Administration patients. Pain Med. 2014;15(11):1911-29. https://doi.org/10. 1111/pme.12480.

24. Harris PA, Taylor R, Minor BL, et al. The REDCap consortium: Building an international community of software partners. J Biomed Inform. 2019;95: 103208. https://doi.org/10.1016/j.jbi.2019.103208.

25. Finch WH, Finch MEH. Regularization methods for fitting linear models with small sample sizes: fitting the Lasso Estimator using R. Pract Assess Res Eval. 2016;21(7):1-13.

26. Littell R, Milliken G, Stroup W, et al. SAS system for mixed models. Cary: SAS Publishing; 1996.

27. Akaike H. A new look at statistical model identification. IEEE Trans Auto Con. 1974;19:716-23.

28. Higgins C, Smith BH, Matthews K. Evidence of opioid-induced hyperalgesia in clinical populations after chronic opioid exposure: a systematic review and meta-analysis. Br J Anaesth. 2019;122(6): e114-26. https://doi.org/10.1016/j.bja.2018.09. 019.

29. Eisenberg E, Suzan E, Pud D. Opioid-induced hyperalgesia $(\mathrm{OIH})$ : a real clinical problem or just an experimental phenomenon? J Pain Symptom Manage. 2015;49(3):632-6. https://doi.org/10. 1016/j.jpainsymman.2014.07.005. 
30. Ram KC, Eisenberg E, Haddad M, et al. Oral opioid use alters DNIC but not cold pain perception in patients with chronic pain-new perspective of opioid-induced hyperalgesia. Pain. 2008;139(2): 431-8. https://doi.org/10.1016/j.pain.2008.05.015.

31. Edwards RR, Dolman AJ, Michna E, et al. Changes in pain sensitivity and pain modulation during oral opioid treatment: the impact of negative affect. Pain Med. 2016;17(10):1882-91. https://doi.org/10. 1093/pm/pnw010.

32. Laigaard J, Bache N, Stottmeier S, et al. Cognitive function during opioid tapering in patients with chronic pain: a prospective cohort study. J Pain Res. 2020;13:3385-94. https://doi.org/10.2147/JPR. S273025.

33. DiBenedetto DJ, Wawrzyniak KM, Finkelman M, et al. Relationships between opioid dosing, pain severity, and disability in a community-based chronic pain population: an exploratory retrospective analysis. Pain Med. 2019;20(11):2155-65. https://doi.org/10.1093/pm/pny240.

34. Murphy JL, Clark ME, Banou E. Opioid cessation and multidimensional outcomes after interdisciplinary chronic pain treatment. Clin J Pain. 2013;29(2):109-17. https://doi.org/10.1097/AJP. 0b013e3182579935.
35. Mackey K, Anderson J, Bourne D, et al. Benefits and harms of long-term opioid dose reduction or discontinuation in patients with chronic pain: a rapid review. J Gen Intern Med. 2020;35(Suppl 3):935-44. https://doi.org/10.1007/s11606-020-06253-8.

36. Kovacs FM, Abraira V, Royuela A, et al. Minimal clinically important change for pain intensity and disability in patients with nonspecific low back pain. Spine (Phila Pa 1976). 2007;32(25):2915-20. https://doi.org/10.1097/BRS.0b013e31815b75ae.

37. Dowell D, Haegerich T, Chou R. No shortcuts to safer opioid prescribing. $\mathrm{N}$ Engl $\mathrm{J}$ Med. 2019;380(24):2285-7.

38. Centers for Disease Control and Prevention. CDC advises against misapplication of the Guideline for Prescribing Opioids for Chronic Pain. 2019. https:// www.cdc.gov/media/releases/2019/s0424-advisesmisapplication-guideline-prescribing-opioids.html. Accessed 17 Aug 2021.

39. Covington EC, Argoff CE, Ballantyne JC, et al. Ensuring patient protections when tapering opioids: consensus panel recommendations. Mayo Clin Proc. 2020;95(10):2155-71. https://doi.org/10. 1016/j.mayocp.2020.04.025. 